Voix et Images

voixetimages

\title{
Du théâtre, du tragique
}

\section{Pierre Hébert}

Volume 20, numéro 1 (58), automne 1994

\section{Saint-Denys Garneau}

URI : https://id.erudit.org/iderudit/201155ar

DOI : https://doi.org/10.7202/201155ar

Aller au sommaire du numéro

\section{Éditeur(s)}

Université du Québec à Montréal

\section{ISSN}

0318-9201 (imprimé)

1705-933X (numérique)

Découvrir la revue

Citer cet article

Hébert, P. (1994). Du théâtre, du tragique. Voix et Images, 20(1), 232-237.

https://doi.org/10.7202/201155ar d'utilisation que vous pouvez consulter en ligne.

https://apropos.erudit.org/fr/usagers/politique-dutilisation/ 
Revue des revues

\section{Du théâtre, du tragique}

Pierre Hébert, Université de Sherbrooke

Nos départements des lettres, à mon grand étonnement d'ailleurs, favorisent certains genres dans la formation des étudiants, au détriment inévitable d'autres pans de la production littéraire. Et je ne parle même pas ici du rapport entre les genres canonisés et les autres plus marginaux: l'on suivra, comme étudiant, quantité de cours sur la prose par exemple, et, à l'occasion, quelques cours sur la poésie ou le théâtre. Cette disproportion est peut-être encore plus grande sur le plan de la formation théorique. Dans le secteur des études littéraires, il semble que ce que l'on veut apprendre l'emporte sur ce que l'on doit apprendre.

Cela dit, il est temps que cette chronique évite l'écueil qu'elle semble si facilement déceler chez d'autres. Voilà pourquoi le théâtre prendra une place importante ici; mais cela tient tout autant à la qualité des numéros de la revue Jeu dont il sera question.

\section{Théâtre-femmes, théâtre tragique}

Bien malin le lecteur qui a vu un lien entre le théâtre-femmes et le théâtre tragique! Il s'agit plutôt de deux numéros sur lesquels je m'attarderai avec l'espoir de transmettre le plaisir que j'ai eu à les découvrir.

Légèrement modifié pour l'occasion, Jeu s'ouvre sur un éditorial désormais engagé, et qui s'intitule "Un mouvement irréversible", par Lorraine Camerlain, rédactrice en chef et directrice de ce numéro particulier, "Théâtre-femmes ${ }^{1}$ ". L'inquiétude qui traverse ce texte liminaire est le premier cri d'un écho qui retentit dans chaque article subséquent. C'est ce qui s'appelle "donner le ton "... Il faut dire que l'accession des femmes, "plus visiblement et en plus grand nombre aux postes clés au moment où le pouvoir est chancelant, dans un état de crise permanent". (p. 10), ne rassure pas: elles prennent le volant d'une bagnole pour le moins amochée. Il ne faudra pas l'oublier si, bientôt, la société tout entière devait tomber en panne. 
Entre autres, pour ouvrir le débat, trois personnes ont accepté de relire le numéro 16 de Jeu paru en 1980; et qui s'intitulait justement "Théâtre-femmes". Lola Noël se demande si, en dépit des changements importants des dernières années, un ordre nouveau a vraiment été instauré; Dominick Parenteau-Lebeuf convient que, dans le déplacement du collectif à l'individuel, "le chemin est [sic] pas fini d'ouvrir, mais on passe la charrue qu'on a choisie dessus" (p. 22). Enfin, Denis O'Sullivan va dans le même sens: "Le féminisme a fait du théâtre une arme efficace quant à la prise de conscience et à l'articulation des revendications. Mais son efficacité est plus relative lorsqu'il s'agit d'entraîner des changements à la base" (p. 25). Les avancées sont importantes, mais les acquis radicaux sont à venir.

Soyons tout de même réalistes: le théâtre féministe ne peut tout recréer. Il n'est pas ce nouvel atlas qui peut supporter tout le poids des changements essentiels. Petite consolation, car cette déception donne aussi la mesure des espoirs qui reposent encore dans le féminisme. Mais que de chemin à parcourir lorsque, ainsi que le signale Diane Godin, la pièce la plus jouée au monde, En attendant Godot, "embrasse l'humanité tout entière par l'emploi du seul masculin ${ }^{2}$ "

Pourtant, ce n'est pas seulement "l'homme synecdoque", celui qui de partie se prend poúr le tout, qui doit être dénoncé; c'est aussi toute image de la femme folle, hystérique, de la sorcière qui est en cause dans cet effort de nouveau regard social. On sait combien la sorcière a été étudiée, et Lynda Burgoyne aborde le personnage féminin, et en particulier la sorcière, dans quelques textes récents: "sorcière un jour... sorcière toujours ", semble-t-elle constater à la lecture de quelques pièces récentes (de Sally Clárk, Brad Fraser et Yukio Mishima).

Cette livraison de "Théâtre-femmes"s" prend surtout la manière d'un bilan, d'une réflexion. Le numéro consacré à la tragédie eșt d'une autre eau: plus ancrées dans l'analyse, les études tendent vers une approche sociologique du statut actuel du tragique.

Michel Vaïs s'est entretenu avec de nombreux metteurs en scène, acteurs, enseignants (Monique Mercure, André Brassard, Marthe Turgeon, Lorraine Pintal, etc.). Cette sorte d'enquête sur "Le jeu tragique $^{3}$ " lance quantité de pistes intéressantes quant à l'intérêt actuel à l'endroit du tragique, de la découverte de l'Autre après le stade du miroir au sentiment de l'effondrement en cette fin de siècle. Chacun livre ses réflexions sur l'art (du) tragique, tant dans les circonstances de son épanouissement que dans son exécution. 
Or, cherchera-t-on une excellente introduction au tragique et aux déplacements qu'il a connus dans l'histoire, jusqu'à l'apparition récente du sida? Alors il faudra lire "Tragique et tragédie ", d'Alexandre Lazaridès.

Depuis Copernic, l'histoire de l'humanité est celle de son humiliation: l'homme n'est plus désormais le centre de l'univers, ni le centre de l'espèce (Darwin) et, avec' Freud, il n'est même plus maître de son propre moi. Le trait commun de cette déchéance, c'est l'irresponsabilité. Or, comme le tragique s'enracine dans la responsabilité et la culpabilité, "nous comprenons tout ce qui rend le concept de tragique étranger à la psyché moderne ${ }^{4}{ }_{n}$. Si le mur est essentiel à la tragédie, comme le posait Ronfard dans l'article précédent, on comprend alors pourquoi le vrai tragique défie l'ordre du monde.

Lazaridès éclaire ainsi le sens (ou l'absence de sens) du tragique, de la catharsis, de l'erreur. Mais il conclut par une réflexion sur le sida comme "forme de fatalité nouvelle, amèrement propice à un sentiment tragique de la condition humaine " (p. 41).

Je signale rapidement une étude de France Bélanger et de Raymond Paul, "Famille et fatalité chez Michel Tremblay: la filiation tragique». Enfin, pour mémoire, Louise Vigeant définit en une dizaine de pages un ensemble de mots clés - catharsis, pathos, culpabilité, etc. - qui en apprendront beaucoup aux inconditionnels du Nintendo, la plus grande simulation d'anti-fatalité ${ }^{5}$.

\section{J'aurais voulu être un artiste...}

...Et pouvoir inventer ma vie, chante Claude Dubois. Mais, au delà des clichés, des mythes, qu'est-ce qu'un artiste?

Possibles réfléchit à la question, et sérieusement. "Artiste; rares sont les mots aussi chargés sémantiquement et qui possèdent des connotations aussi disparates ${ }^{6}$, écrit Alexandra Jarque dans l'éditorial. Comment l'Artiste, aujourd'hui, se définit-il par rapport à la société? Choisissez votre réponse: l'albatros ridiculisé, le missionnaire laïque, le réformateur social. Et; pourquoi pas, le désabusé. Réflexions, tables rondes avec de jeunes auteurs, essais, analyses composent le faisceau d'opinions qui donnent de l'artiste cette image forcément multiple car, là plus qu'ailleurs, l'unanimité a mauvais goût.

Je me permets de retenir, cependant, l'essai de Terry Archibald et de Diane Perron, "De Léonard à Madonna", dont le sous-titre dévoile les vraies couleurs: "ou ce qu'il advient de l'artiste dans la société postmoderne": Dans ce nouveau monde, l'artiste n'est plus ce phare 
qui balaie les récifs dont parlait, si je me rappelle bien, Victor Hugo, car pareille image le réduirait à un seul point d'où part la lumière. Non, tout autre est désormais la contribution de l'artiste, dont l'œil a éclaté en des milliers de cellules visuelles qui se sont greffées aux modes mineurs et majeurs de la culture, aux sphères du privé et du public, à l'utilitaire et au fonctionnel. "L'art est partout " mais, lourd tribut, l'artiste n'est nulle part:

Nous ne sommes plus à l'ère de l'homme révolté devant l'absurdité, l'absence de sens de la création, nous sommes à celle de l'homme survolté - survolté de.signes, de mots, de sonorités, d'images, de symboles, de significations. Et, quoi qu'on en dise, de sens. Que l'Artiste accepte une fois pour toutes qu'il ne sera pas un Léonard des temps modernes. Personne ne lui demande plus de réinventer le monde et d'engager l'avenir dans la moindre de ses œuvres (p. 94).

Le rôle de l'artiste se dissout ainsi avec l'éclatement des lieux du sens. Mais la multiplication des signes engendre aussi ce que j'appellerais volontiers le règne de "La Grande Équivalence». Umberto Eco a déjà noté que la censure ne se manifeste plus aujourd'hui par rétention ou exclusion, mais par profusion. À défaut d'espaces sémantiques à haute densité, le sens éparpillé perd en force ce qu'il gagne en dissémination. Il est tentant, par un syllogisme un peu boiteux, de rattacher ce sentiment de perte au sens du tragique dont il a été question plus haut et, particulièrement, à l'humiliation humaine des derniers siècles. L'artiste vit lui aussi cette humiliation, ressentie comme la perte d'un pouvoir (pour peu qu'il en ait eu un), perte mesurée par la fragmentation de sa parole diluée dans les multiples manifestations sociales du sens ${ }^{7}$.

\section{Lectures diverses}

Je renonce, en guise de troisième volet de cette chronique, à toute unité, à ses pompes et à ses œuvres. Comme l'artiste, le chroniqueur tiendra des propos fragmentés touchant l'un ou l'autre domaine de la recherche en littérature québécoise.

Je saisis d'abord, en un seul paragraphe, trois numéros de Mcebius, qui mériteraient certainement beaucoup plus. D'abord, dans une étude intéressante, à l'écriture enlevée, Jean-Marc Lemelin propose "Quatre pistes de lecture de Volkswagen Blues ${ }^{8}$. Écrit comme d'un seul trait, l'article trouve son point culminant, ce me semble, dans la section "La piste du nom propre ou la quête de l'identité". En second lieu, le numéro suivant étale un ensemble de propos autour de "La résistance à l'écriture" qui, à sa manière, recoupe presque par nécessité le tragique et le statut de l'artiste: "Il convient de chanter 
avec réserve à l'époque de la dévastation du monde ${ }^{9} . "$ Enfin, le troisième numéro s'intitule "Écrivains-paroliers ${ }^{10}$ ", une série de réflexions et d'études, dont une sur Ducharme et Mistral par Sylvain Lambert. Mais, aussi dans ce numéro, l'un des textes'le plus triste, j'allais dire le plus tragiquement vrai que j'aie lus: "Le piège", de Raymond Lévesque. Je n'en dis pas davantage, lisez-le plutôt, "si la vie vous intéresse "...

Bien sûr, nos auteurs habituels sont toujours bien fréquentés: Marie-Claire Blais ${ }^{11}$, Carrier et Tremblay ${ }^{12}$. Quant à Victor-Lévy Beaulieu, il se voit consacrer un numéro tout entier par Tangence ${ }^{13}$. En outre, puisqu'il est question de thématique approfondie, Canadian Issues/Thèmes canadiens consacre quelque deux cents pages aux "Voyages, réels et imaginaires, personnels et collectifs", étudiés par une douzaine d'analystes et de critiques ${ }^{14}$. Enfin, un nouveau venu qui traitera à l'occasion de littérature québécoise: Les Cabiers d'bistoire du Québec au $x x^{e}$ siècle ${ }^{15}$. Ainsi, ce premier numéro aborde Le Devoir comme éditeur littéraire et offre plusieurs inédits de SaintDenys Garneau.

1. Cabiers de théâtre Jeu, "Thêâtre-femmes ", nº 66, mars 1993.

2. Diane Godin, "C'est toute l'humanité", ibid., p. 27.

3. Michel Vaïs, "Le jèu tragique", Cabiers de théâtre Jeu, no 68, septembre 1993 , p. 10-30.

4. Alexandre Lazaridès, "Tragique et tragédie", ibid., p. 35.

5. Notons en outre une bibliographie sur le tragique de Monique Forest. Ces propos sur le théâtre pourront être enrichis par un dossier substantiel dans Nuit blanche, "Le théâtre avec ou sans texte ", no 55, mars-avril-mai 1994, p. 44-71, et par Protée, "Gestualités ", vol. XXI, n 3, automne 1993.

6. Alexandra Jarque, "Éditorial ", Possibles, vol. XVIII, $\mathrm{n}^{\circ} 1$ ("L'Artiste. Autoportraits"), hiver 1994, p. 7.

7. On pourra ajouter à cette lecture la très riche réflexion de Fernand Dumont, "L'intellectuel et le citoyen", parue plus tôt (Possibles, vol. XVII, n ${ }^{\text {os }} 3-4$, étéautomne 1993, p. 319-333). En outre, puisque le numéro de Possibles s'intitule "Autoportraits", je signale, plus par contiguité d'appellation, "Le récit de soi ", Tangence, $\mathrm{n}^{\circ} 42$ (décembre 1993).

8. Jean-Marc Lemelin, "Quatre pistes de lecture de, Volkswagen Blues", Mobbius, $\mathrm{n}^{\circ}$ 57, automne 1993, p. 101-116.

9. Paul Chamberland, "L'heure est très sévère", Mcebius, n 58 ("La résistance à l'écriture $n$ ), hiver 1993, p. 47.

10. Moebius, n० 59, "Écrivains-paroliers", hiver 1994.

11. Dominique Perron, "Les discours sociaux dans Les Nuits de l'Underground de Marie-Claire Blais ", Canadian Literature, $\mathrm{n}^{\text {os }} 138-139$, automne-hiver 1993, p. 5370; Marie Couillard, "Visions d'Anna ou l'écriture du vertige de Marie-Claire' Blais "Québec Studies, no 17, automne 1993-hiver 1994, p. 117-124; dans le même numéro, Karin $M$. Egloff, "Entre la mère-miroir et l'amer voir: le regard écorché dans La Belle Bête de Marie-Claire Blais *, p. 125-133. Le numéro fournit aussi deux études sur les années trente et quarante, en l'occurrence sur un rapprochement entre La Chair décevante et La Convention (Fernand Roy), Au pied de la pente 
douce (Victor-Laurent Tremblay). Notons aussi, toujours dans cette livraison, * $L a$ Maison du remous de Nicole Houde, ou le roman de la terre au féminin ", de Lori Saint-Martin, p. 187-199.

12. Danielle Forget, "Les paradoxes de la langue chez Roch Carrier et Michel Tremblay ", Études littéraires, vol. XXVI, nº 3, hiver 1993-1994, p. 119-133.

13. Tangence, $\mathrm{n}^{\circ} 41$ («Interdiscursivité dans l'œuvre de Victor-Lévy Beaulieu»), octobre 1993.

14. "Voyages réels et imaginaires, personnels et collectifs", Canadian Issues/Thèmes canadiens, vol. XVI, 1994.

15. Les Cabiers d'bistoire du Québec au XX siècle, $\mathrm{n}^{\circ} 1$ (hiver 1994). Les articles sur Le Devoir sont du signataire de cette chronique et de Patrick Nicol, et les inédits sont présentés par Giselle Huot. 